\title{
CORRELAÇÃO ENTRE O DIÂMETRO DO FOLÍCULO PRÉ-OVULATÓRIO E A EFICIÊNCIA REPRODUTIVA EM VACAS Bos taurus indicus SUBMETIDAS À INSEMINAÇÃO ARTIFICIAL EM TEMPO FIXO
}

\author{
(Correlation between preovulatory follicle diameter and reproductive efficiency in \\ Bos taurus indicus cows submitted to timed artificial insemination)
}

\author{
Danilo Amadori Martins de Oliveira, Luiz Ernandes Kozicki, Rafaela Talini, Victor Breno \\ Pedrosa, Grassiele Gassenferth, Romildo Romualdo Weiss ${ }^{1}$ \\ ${ }^{1}$ Correspondência: rrweiss@bol.com.br
}

RESUMO: O presente estudo objetivou avaliar o diâmetro do folículo ovariano préovulatório (FPO) no momento da IATF em vacas multíparas Bos taurus indicus correlacionando-o com a taxa de concepção (TC). Foram utilizadas 145 vacas, paridas até 65 dias, e submetidas a único protocolo: no dia 0 (d0) implante de 1,0 g de progesterona + aplicação de 2,0 mg de benzoato de estradiol; em d8 remoção dos implantes e aplicação de $500 \mu \mathrm{g}$ de D-Cloprostenol + $300 \mathrm{UI}$ de Gonadotrofina coriônica equina e 1,0 mg de cipionato de estradiol; no d10 mensurou-se o FPO (ultrassom) e procedeu-se a IATF com sêmen congelado. No d35 pós IATF realizou-se o diagnóstico de prenhez. A taxa de concepção à IATF foi de $62,7 \%$ e 0 diâmetro médio do FPO nas vacas prenhes foi de $15,1 \mathrm{~mm}$, e de $13,7 \mathrm{~mm}$ nas vazias $(\mathrm{P}=0,03)$. Foram estabelecidos 04 estratos relativos ao diâmetro dos FPOs $(\mathrm{mm})$ : 8,0 a 11,$0 ; 11,1$ a 14,$0 ; 14,1$ a 17,0 e 17,1 a 20,0, resultando respectivamente em 37,$0 ; 75,7 ; 66,6$ e $72,7 \%$ de TC. Estratificou-se ainda o diâmetro do FPO em $\leq 11,0$ $\mathrm{mm}$ e $\geq 11,1$ resultando em TC de $36,8 \%$ e $72,0 \%(P<0,01)$ respectivamente. Onze animais ovularam antes da IATF, com TC de 36,4\%. Concluiu-se que 0 diâmetro do FPO na IATF tem correlação com a TC; FPOs > 11,1mm constituem forte indicativo de maior eficiência reprodutiva.

Palavras-chave: diâmetro folículo pré-ovulatório; taxa de concepção; IATF; vacas Bos taurus indicus; multíparas

ABSTRACT: The present study aimed to evaluate the diameter of the ovarian preovulatory follicle (POF) at the time of TAl in multiparous cows Bos taurus indicus correlating it with the conception rate (CR). POR Extenso o 145 cows were used, calving to 65 days and subjected to a single protocol: day 0 (d0) implantation of 1.0 $\mathrm{g}$ of progesterone + application $2 \mathrm{mg}$ of oestradiol benzoate; d8 removal P4 implant and applied to $500 \mathrm{mcg}$ of D-cloprostenol + $300 \mathrm{IU}$ of equine chorionic gonadotropin, and $1.0 \mathrm{mg}$ estradiol cypionate; d10 measured the POF (ultrasound) and proceeded to TAI with frozen semen. On d35 after TAl the pregnancy diagnosis was performed. The conception rate to TAI was $62.7 \%$ and the average POF on the pregnant cows was $15.1 \mathrm{~mm}$ and $13.7 \mathrm{~mm}$ on the open cows $(P=0.03)$. Related to diameter of the POF, four diameter ranges were established: 8.0 to $11.0 \mathrm{~mm} ; 11.1$ to $14.0 \mathrm{~mm} ; 14.1$ to $17.0 \mathrm{~mm}$ and 17.1 to $20.0 \mathrm{~mm}$, resulting respectively in $37.0 ; 75.7 ; 66.6$ and $72.7 \%$ of CR. The POF's diameters were also distributed in $\leq 11.0 \mathrm{~mm}$ e $\geq 11.1$ resulting in $36.8 \%$ and $72.0 \%$ CR $(P<0.01)$ respectively. Eleven animals ovulated before TAI with CR of $36.4 \%$. It was concluded that the POF's diameter at TAI has 
correlation with CR; FPOs $>11.1 \mathrm{~mm}$ are strong indicatives of greater reproductive efficiency.

Key Words: preovulatory follicle diameter; conception rate; TAI; Bos taurus indicus cows; multiparous 


\section{INTRODUÇÃO}

A inseminação artificial (IA) é uma tecnologia utilizada em todo o mundo, auxiliando principalmente no melhoramento genético de rebanhos de várias espécies de animais, com ênfase aos bovinos. No Brasil, o percentual de fêmeas bovinas inseminadas artificialmente ainda é baixo, atingindo 12,0\% em 2015 (Baruselli et al., 2016). As dificuldades na observação do estro constituem-se nos principais fatores limitantes da utilização da IA, principalmente em fêmeas de origem zebuína, as quais apresentam estros de duração mais curta, além de que 50,0\% desses animais manifestam o estro em períodos noturnos (Ereno et al., 2007), reduzindo ainda mais a eficiência reprodutiva.

A inseminação artificial em tempo fixo (IATF), constitui em biotecnologia, que elimina a necessidade de observação de cio, tornando mais fácil o emprego da IA, acelerando desta forma - ganho genético dos rebanhos mediante $O$ emprego de material genético superior. A IATF atingiu os limites de aproximadamente 10,5 milhões de procedimentos em 2015, um crescimento de $11,2 \%$ comparado ao ano anterior num levantamento baseado no número de protocolos comercializados por empresas do setor e doses de sêmen comercializadas (Baruselli, 2016). Apesar disso, nos últimos anos as taxas de prenhez de fêmeas submetidas aos protocolos de IATF, estabilizou-se ao patamar de 40 a $60 \%$. (Meneghetti et al., 2009; Sá Filho et al., 2009; Souza et al., 2016).

Vários fatores podem influenciar o sucesso de programas de IATF em bovinos (corte e leite). Dentre eles, insere-se a concentração e a dose dos hormônios utilizados para a sincronização da ovulação, bem como o diâmetro do folículo dominante (FD) no momento da inseminação artificial
(Pfeifer et al., 2015). O diâmetro do FPO no momento da IATF, destaca-se como um importante fator de sucesso nas taxas de concepção, onde folículos com maiores diâmetros, contém maiores concentrações de estradiol e maior possibilidade de ovulação, resultando em aumento nos índices de fertilidade em rebanhos sincronizados (Sá Filho et al., 2010). Além disso, um folículo com maior diâmetro origina um corpo lúteo de maior dimensão, elevando consequentemente as possibilidades de manutenção da prenhez (Lonergan et al., 2013). As células da teca interna e da granulosa, (componentes do folículo), se transformam em células luteínicas pequenas e grandes, respectivamente, desenvolvendo 0 corpo lúteo (Giometti et al., 2009); portanto, quanto maior for o FPO, maior será o aporte de células luteínicas na cratera de ovulação, acarretando maior dimensão do CL e, consequentemente, maior produção de $\mathrm{P} 4$.

O objetivo do presente estudo foi avaliar o diâmetro do folículo préovulatório no momento da inseminação artificial em fêmeas Bos taurus indicus, submetidas a um protocolo de IATF e correlacionar com as taxas de concepção.

\section{MATERIAL E MÉTODOS}

Foram utilizadas 145 vacas multíparas de corte Bos taurus indicus lactantes, com período de 40 a 65 dias de dias abertos, procedentes de uma fazenda comercial. As vacas eram criadas em sistema extensivo, com suplementação mineral e água ad libitum, apresentando ao início da aplicação do protocolo escore de condição corporal (ECC) entre 2,5 e 3,5 ( 1 = magra; 5 = obesa) (Houghton et al., 1990).

Todas os animais foram submetidos a um protocolo de sincronização de estro e inseminadas artificialmente em tempo fixo com sêmen congelado de touro 
fértil, na estação reprodutiva. Ao início da administração do protocolo hormonal (d0) (diagrama figura 1) as vacas receberam um implante de $\mathrm{P} 4+$ benzoato de estradiol (BE); no d8 foi retirado $o$ implante $e$ aplicado $D$ Cloprostenol + gonadotrofina coriônica equina + cipionato de estradiol. No d10 procedeu-se à mensuração do folículo pré-ovulatório (FPO) mensurando-se o diâmetro maior + diâmetro menor $\square$ por 2 (Gastal et al., 2008), mediante aparelho de ultrassom (Mindray DP 2200 vet, transdutor linear de frequência de $6 \mathrm{MHz}$; China) + a IATF com sêmen congelado. Os diâmetros dos FPO foram estratificados em várias faixas de dimensão como segue: 8,0 a 11,0; 11,1 a $14 ., 0 ; 14,1$ a 17,0 e 17,1 a $20,0 \mathrm{~mm}$ (Ribeiro Filho et al., 2013). O diagnóstico de prenhez (DP) foi realizado 35 dias após a IATF (d45) visando a taxa de concepção. A análise do sêmen utilizado obedeceu às Normas do CBRA (1998).

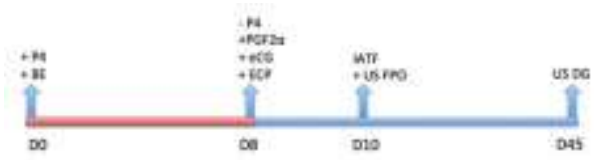

Figura 1 - Prototolo nomonal. Impiarse P4 $11.0 \mathrm{~g}$. Cronipres Mono Desed). Biogenesis lago, Curtiba - Brasil]; BE [2.0 mg, Bloestrogonn, Blogonesis Bagol; D.

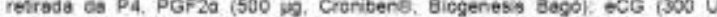
Ecegonk, Biogeness Bagoi ECP (1,0 mg, Croni-Cipls, Blogenesis Bago): D10-1ATF + exame utrassonografico pata mensuraç bo do PPO; D45 - DG.

Análise estatística

As medidas de diâmetro dos FPO foram estratificados em 4 categorias de intervalos $(8,0$ a 11,$0 ; 11,1$ a 14,$0 ; 14,1$ a 17,0 e 17,1 a $20,0 \mathrm{~mm})$. Para cada uma das classes de FPO verificou-se a respectiva taxa de concepção. Assim os dados de diâmetro dos FPO versus dados da taxa de concepção (TC) foram estatisticamente tratados empregandose o teste de correlação de Pearson. Em posterior análise, outra estratificação foi feita (tabela 2) restringindo-se 0 intervalo de dimensão de folículos para $\square 11,0$ e $\square 11,1 \mathrm{~mm}$ e correlacionando-se as duas variáveis. Para checar as diferenças das TC entre os diferentes intervalos do diâmetro do FPO foi utilizado o teste Exato de Fisher. $O$ nível de significância empregado foi de $\mathrm{P}<0,05$. As correlações foram efetuadas baseandose nos critérios descritos por Mukaka (2012). As médias de cada categoria de FPO foram executadas pelo teste de Tukey. O programa do software GraphPad Prism, versão 5.0 (Prism 5 Statistics Guide, 2007, GraphPad Software Inc., San Diego CA.) foi utilizado para a análise estatística.

\section{RESULTADOS E DISCUSSÃO}

Das 145 vacas inseminadas, 91 tornaram-se prenhes resultando em TC geral na IATF de $62,7 \%$ (Tabela 1 ). Onze vacas haviam ovulado até o dia da IATF e não foram contabilizadas para o diâmetro de FPO. Na correlação entre as categorias do diâmetro do FPO e a TC verificou-se que FPO entre 11,1 e $14,0 \mathrm{~mm}$ resultaram em maior TC seguido da categoria $\geq 17,1 \mathrm{~mm}$. $\mathrm{Na}$ estratificação das categorias de $\leq 11,0$ e $\geq 11,1 \mathrm{~mm}$ versus TC detectou-se diferenças de TC entre esses dois grupos $(P<0.05)$ (Tabela 2$)$. A média do FD das vacas gestantes foi de $15,1 \mathrm{~mm}$ e das não gestantes de $13,7 \mathrm{~mm}$.

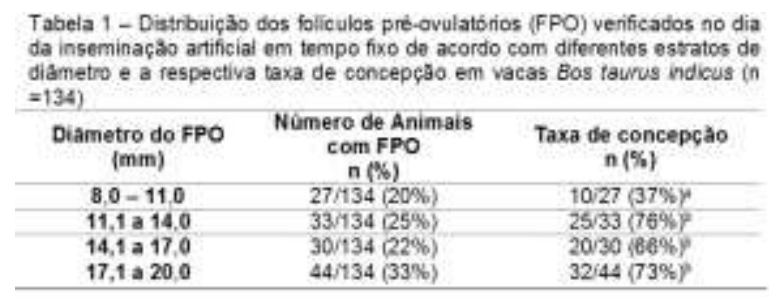


Tabeia 02 - Diámetro do foliculo pré-ovulatorio versus a taua de concepp̧ăo entre grupos de vacas Bos taurus indicus, estrinticados em duas faxas com $<11.0$ 11.1, submefidos a protocolos de inseminaçăo artificial em tempo fixo in $=145$ )

\begin{tabular}{|c|c|}
\hline $\begin{array}{c}\text { Diametro do FD } \\
(\mathrm{mm})\end{array}$ & $\begin{array}{c}\text { Taxa de concepção } \\
\mathrm{n}(\%)\end{array}$ \\
$\mathbf{5 1 1 , 0}$ & $10 / 27(37 \%)$ \\
$\mathbf{2 1 1 , 4}$ & $77 / 107(725)$ \\
\hline Valor de P & 0,008 \\
\hline
\end{tabular}

Obs.: Letras diferentes na mesma coluna indica significância ao nível de $\mathrm{P}<0,002$.

Em estudo sobre a redução das dimensões de um folículo pré-ovulatório, Vasconcelos et al. (2001) verificaram que a ovulação de pequenos folículos acarretava menores taxas de fertilidade, devido ao desenvolvimento de menor corpo lúteo e consequentemente menor concentração de progesterona circulante. $\mathrm{O}$ diâmetro do maior folículo ovariano teve significativa influência sobre a taxa de prenhez à IATF, porquanto à medida em que as dimensões do folículo eram maiores, maior era a taxa de prenhez (Sá Filho et al., 2010). O presente estudo mostrou que menores dimensões do FPO (folículos $\leq$ que $11,1 \mathrm{~mm}$ ) acarretaram menor TC. Ao se observar as categorias dos diâmetros do FPO versus prenhez, interessantemente os folículos com diâmetros > 11,1 a 14,0 mm detiveram o maior percentual de TC que os demais, embora sem significância. De modo geral os folículos com diâmetros maiores que $11,1 \mathrm{~mm}$ resultaram em maior TC (Tabela 1), corroborando os relatos dos pesquisadores (Sá Filho et al.,2010)

Diversos são os fatores influentes sobre o sucesso da IATF em vacas Bos taurus indicus, dentre os quais pode-se citar o local, o rebanho, a paridade e o escore da condição corporal dos animais (Bó et al., 2007; Sá Filho et al., 2009). Estudos em novilhas (Perry et al., 2007) e em vacas multíparas, relatam a influência da dimensão do folículo ovulatório sobre a TC (Perry et al., 2005; Meneghetti et al., 2009). Acredita-se que haja uma correlação entre as dimensões do FPO e a TC no fato, de que folículos menores tendem a produzir inferiores quantidades de estradiol no momento da ovulação, devido ao menor número de células da granulosa e da teca interna que apresentam (Vasconcelos et al., 2001). Isso afetaria o feedback positivo para a liberação adequada do $\mathrm{LH}$, gerando menor comprometimento da ovulação e da TC (Sá Filho et al. 2010). Estudos demonstraram que há uma proporção entre o diâmetro do FPO e a concentração de estradiol em vacas (Sá Filho et al., 2009). Além disso, vacas ao apresentarem um FPO de maior diâmetro exibirão maior quantidade de estradiol, promovendo modificações no meio-ambiente uterino, otimizando o transporte espermático e beneficiando a concepção (Sá filho et al., 2010), não ocorrendo esta condição em casos de insuficientes concentrações estrogênicas (Hawk, 1983).

A expressão gênica de receptores de $\mathrm{LH}$ nas células da granulosa é outro importante fator que contribui para o sucesso da TC, uma vez que quanto maior a quantidade de receptores, maior será a resposta à gonadotrofina (crescimento e maturação folicular final) (Dias et al., 2009). A maior expressão de receptores em fêmeas zebuínas ocorre quando o folículo atinge o diâmetro de aproximadamente 7,0 $\mathrm{mm}$, aumentando este número de acordo com o aumento do diâmetro folicular (Simões et al., 2012).

No presente estudo, a estratificação em duas categorias de dimensões de FPO $(<11,0$ e $>11,1$ $\mathrm{mm})$ e confrontando com a TC, evidenciou que as dimensões do FPO exercem significativa influência sobre a eficiência reprodutiva (Tabela 2). Borsato et al. (2004) ao correlacionarem as TC em novilhas Bos taurus taurus $\mathrm{x}$ Bos taurus indicus com as dimensões dos FPO, verificaram TC de 11,1; 63,8 e $88,2 \%$ para folículos de $7-10 \mathrm{~mm}, 10-13$ 
$\mathrm{mm}$ e de 13-19 $\mathrm{mm}$ respectivamente. Em estudo com vacas Nelore Silveira et al. (2014) observaram que a dimensão média dos FPO foi de $12,4 \mathrm{~mm}$, sendo de 13,2 mm nas fêmeas gestantes, e de $10,9 \mathrm{~mm}$ nas vazias. Perry et al. (2005), utilizaram $\mathrm{GnRH}$ (indutor da ovulação), e obtiveram resultados muito similares aos do corrente estudo, verificando menores TC em animais com FPO < $11,0 \mathrm{~mm}$. Resumidamente há uma correlação positiva entre o diâmetro do FPO e a eficiência reprodutiva (Silveira et al., 2014; Perry et al., 2005) características estas também observadas no presente estudo.

Além desses aspectos, estudos comprovam que animais com um maior diâmetro de FPO possuem maior capacidade de manutenção da prenhez, porquanto, folículos de maiores diâmetros originam CLs com maiores diâmetros, gerando maiores quantidades de P4 produzida e reduzindo as perdas embrionárias (Santos, 2004). Siqueira et al. (2009) constaram elevada correlação entre o volume de tecido luteal $e$ as concentrações de P4. Mann e Lamming (2001) afirmam que baixos níveis de P4 prejudicam 0 desenvolvimento embrionário (comprometimento da capacidade do embrião de produzir interferon-tau) com vistas ao reconhecimento da prenhez, assim como favorecem o mecanismo luteolítico.

No presente estudo 11 vacas encontravam-se ovuladas no dia da IATF (7,6\% do total), atingindo somente $36,4 \%$ de prenhez à IATF $(\mathrm{P}<0,05)$. Esses animais ovularam precocemente ou devido a presença de grandes FPO (maior numero de receptores ao $\mathrm{LH}$ ) ou devido a que já na retirada da $\mathrm{P} 4$ tais folículos dominantes encontravam-se em adiantado estágio de desenvolvimento. Assim a administração de eCG ou de ECP podem ter exercido efeitos de sinergismo, acelerando 0 desenvolvimento folicular, maximizando o número de receptores ao $\mathrm{LH}$ e acelerando o processo da ovulação propriamente dito. Isso foi significativamente relevante pois acarretou baixos níveis de TC na IATF. Para esses animais a IATF foi executada tardiamente, período de tempo em que o oócito já não tinha mais viabilidade (Walker et al.1996), porquanto a ovulação se dá em média $27,6 \pm 5,4$ horas após o início do estro. É também factível ponderar a concretização da fertilização dos oocitos dos animais precocemente ovulados, mas estando o oocito já envelhecido, provavelmente comprometeu as clivagens sucessivas, proporcionando baixas TC (Revah e Butler, 1996).

Pursley et al. (1997) e Baruselli et al. (2007), afirmam que uma das possibilidades de redução da variação do momento da ovulação em bovinos (exemplo ovulação precoce), é o emprego de protocolos de IATF, permitindo a execução da IATF em um horário ótimo e consistente com a ovulação. A utilização do ECP sincronizada à remoção do implante de P4, leva a uma ovulação sincronizada dos animais (média de 70 horas após a remoção da P4) (Reis et al., 2004; Martins et al., 2005). A IATF propriamente dita pode ser realizada em um intervalo de 48 a 58 horas após a remoção do implante de P4 (Ayres et al., 2008). Contudo no presente estudo os animais ovularam antes mesmo das 48 horas após a remoção da P4 e da aplicação do eCG e do ECP. Isso comprometeu a TC das vacas ovuladas antes da IATF.

\section{CONCLUSÃO}

A mensuração do diâmetro do FPO no momento da IATF em vacas Bos taurus indicus mostrou-se um 
importante instrumento, revelando que quanto maior a dimensão do FPO maior correlação haverá com a TC; FPOs maiores que $11,1 \mathrm{~mm}$ no momento da IATF, são indicativos de maior eficiência reprodutiva, constituindo-se em importante característica aos profissionais.

\section{NOTAS INFORMATIVAS}

\section{Reservado ao parecer CEUA.}

\section{REFERÊNCIAS}

AYRES, H.; MARTINS, C.M.; FERREIRA, R.M. et al. Effect of timing of estradiol benzoate administration upon synchronization of ovulation in suckling Nellore cows (Bos indicus) treated with a progesterone-releasing intravaginal device. Animal Reproduction Science, v. 109, p. $77-78,2008$.

BARUSELLI, P.S.; GIMENES, L.U.; SALES, J.N.S. Fisiologia reprodutiva de fêmeas taurinas e zebuínas. Revista Brasileira de Reprodução Animal, v.31, p. $205-211,2007$.

BARUSELLI, P.S.; COLLI, M.H.A.; REZENDE, R.G. et al. Situação atual, desafios e perspectivas da reprodução programada em bovinos de corte e de leite. In: SIMPÓSIO INTERNACIONAL DE REPRODUÇÃO ANIMAL APLICADA, 7., 2016, Londrina, Anais...Londrina: SIRAA 2016, p. 163 - 205.

BARUSELLI, P.S. IATF supera dez milhões de procedimentos em amplia o mercado de trabalho. Revista CFMV, v. 69, p. 57 - 60, 2016.

BÓ, G.A.; CUTAIA, L.; PERES, L.C. et al. Technologies for fixed-time artificial insemination and their influence on reproductive performances of Bos indicus cattle. Society for Reproduction and Fertility, v. 64, p. 223 - 236, 2007.

BORSATO, E.A.; LUDWIG JR., H.E.; RUBIN, K.C.P. et al. Relação entre o tamanho do folículo ovulatório e taxa de concepção em novilhas Bos taurus $x$ Bos indicus submetidas a inseminação artificial em tempo fixo. Revista Brasileira de Reprodução Animal, v.28, n.3, p. 137 - 142, 2004.

COLÉGIO BRASILEIRO DE REPRODUÇÃO ANIMAL - CBRA. Manual para exame andrológico e avaliação de sêmen animal. Belo Horizonte: CBRA, 1998. 49 p.
DIAS, C.C.; WECHSLER, F.S.; DAY, M.L. et al. Progesterone concentrations, exogenous equine chorionic gonadotropin, and timing of prostaglandin F2 $\alpha$ treatment affect fertility in postpuberal Nellore heifers. Theriogenology, v.72, p. $378-385,2009$.

ERENO, R.L.; BARREIROS, T.R.R.; SENEDA, M.M. et al. Taxa de prenhez de vacas Nelore lactantes tratadas com progesterona associada à remoção temporária de bezerros ou aplicação de gonadotrofina coriônica equina. Revista Brasileira de Zootecnia, v.36, n.5, p. 1288 1294, 2007.

GASTAL, E.L.; NEVES, A.P.; MATTOS, R.C. et al. Miniature ponies: 1. Follicular, luteal and endometrial dynamics during oestrus cycle. Reproduction, Fertility and Development, v.20, p. $376-385,2008$

GIOMETTI, I.C.; CASTILHO, A.C.S.; SÁ FILHO, O.G. et al. Controle local e endócrino do desenvolvimento e da regressão do corpo lúteo bovino. Revista Brasileira de Reprodução Animal, v.33, p. $34-52,2009$.

HAWK, H.W. Sperm survival and transport in the female reproductive tract. Journal of Dairy Science, v.77, p. 2738 - 2744, 1983.

HOUGHTON, P.L; LEMENAGER, R.P; MOSS, G.E. et al. Prediction of postpartum beef cow body composition using weight to height ratio and visual body condition score. Journal of Animal Science, v.68, p. 1428 - 1437, 1990.

LONERGAN, P.; O`HARA, L.; FORDE, N. Papel da progesterona do diestro na função endometrial e desenvolvimento do concepto em bovinos. Animal Reproduction, v. 10, n.3, p.119 $-123,2013$

MANN, G.E.; LAMMING, G.E. Relationship between maternal endocrine environment, early embryo development and inhibition of the luteolytic mechanism in cows. Reproduction, v.121, p. 175 - 180, 2001.

MARTINS, C.M.;CASTRINI, E.S.C.; SÁ FILHO, M.F. et al. Dinâmica folicular de vacas nelore tratadas com cipionato ou benzoato de estradiol em protocolos de inseminação artificial em tempo fixo. Acta Scientiae Veterinariae, v.33, p. 285, 2005.

MENEGHETTI, M.; SÁ FILHO, O.G.; PERES, R.F.G. et al. Fixed-time artificial insemination with estradiol and progesterone for Bos indicus cows I: Basis for development of protocols. Theriogenology, v.72, n.2, p. 179 - 189, 2009.

MUKAKA, M.M. A guide to appropriate use of Correlation coefficient in medical research. 
Malawi Medical Journal, v.24, n.3, p. $69-71$, 2012.

PERRY, G.A.; SMITH, M.F.; LUCY, M.C. et al. Relationship between follicle size at insemination and pregnancy success. Proceedings of the National Academy of Sciences of the United States of America, v.102, n.14, p. 5268 - 5273, 2005.

PERRY, G.A; SMITH, M.F; ROBERTS, A.J. et al. Relationship between size of the ovulatory follicle and pregnancy success in beef heifers. Journal of Animal Science, v.85, p. 684 - 689, 2007.

PFEIFER, L.F.M.; CASTRO, N.A.; MELO, V.T.O. et al. Timed artificial insemination in blocks: $A$ new alternative to improve fertility in lactating beef cows. Animal Reproduction Science, v.163, p. $89-96,2015$.

PURSLEY, J.R.; KOSOROK, M.R.; WILTBANK, M.C. Reproductive management of lactating dairy cows using synchronization of ovulation. Journal Dairy Science, v.80, n.2, p. $301-306$, 1997.

REIS, E.L.; GIMENES, L.U.; MARQUES, M.O. et al. Efeitos do cipionato e do benzoato de estradiol na dinâmica folicular e luteínica de vacas nelore. Acta Scientiae Veterinariae, v. 32, p. 236, 2004.

REVAH, I.; BUTLER, R. Prolonged dominance of follicles and reduced viability of bovine oocytes. Journal of Reproduction and Fertility, v.106, p. $39-47,1996$.

RIBEIRO FILHO, A.L.; FERRAZ, P.A.; RODRIGUES, A.S. et al. Diâmetro do folículo no momento da inseminação artificial em tempo fixo e taxa de concepção em vacas Nelore. Ciência Animal Brasileira, v.14, n.4, p. 501 - 507,2013.

SÁ FILHO, O.G.; MENEGHETTI, M.; PERES, R.F.G. et al. Fixed-time artificial insemination with estradiol and progesterone for Bos indicus cows II: Strategies and factors affecting fertility. Theriogenology, v.72, p. $210-218,2009$.

SÁ FILHO, M.F.; CRESPILHO, A.M.; SANTOS, J.E.P. et al. Ovarian follicle diameter at timed insemination and estrous response influence likelihood of ovulation and pregnancy after estrous synchronization with progesterone or progestin-based protocols in suckled Bos indicus cows. Animal Reproduction Science, v.120, p. $23-30,2010$.

SANTOS, J.E.P.; THATCHER, W.W.; CHEBEL, R.C. et al. The effect of embryonic death rates in cattle on the efficacy of estrus synchronization programs. Animal Reproduction Science, v.82 83, p. $513-535,2004$.
SILVEIRA, R.O.; SANTOS, G.M.; SILVEIRA, C.O. et al. Avaliação do tamanho do folículo ovulatório e da taxa de concepção de vacas Nelore em protocolos de IATF. In: SIMPÓSIO DE PÓS-GRADUAÇÃO EM ANÁLISES CLÍNICAS, 6., 2014, Viçosa. Anais... Viçosa: SIMPAC, 2014. p.191 - 196.

SIMÕES, R.A.L.; SATRAPA, R.A.; ROSA, F.S. et al. Ovulation rate and its relationship with follicle diameter and gene expression of the LH receptor (LHR) in Nellore cows. Theriogenology, v.77, p. $139-147,2012$.

SIQUEIRA, L.G.; TORRES, C.A.A.; AMORIN, L.S. et al. Interrelationships among morphology, echotexture, and function of the bovine corpus luteum during the estrous cycle. Animal Reproduction Science, v.115, p. 18 - 29, 2009.

SOUZA, A.L.B.; SEGUI, M.S.; KOZICK, L.E. et al. Impact of Equine Chorionic Gonadotropin Associated with Temporary Weaning, Estradiol Benzoate, or Estradiol Cypionate on Timed Artificial Insemination in Primiparous Bos Indicus Cows. Brazilian Archives of Biology and Technology, v.59, e16150389, 2016. Disponível em: http://revistas.bvs-vet.org.br/ Acesso em: 12/09/2016.

VASCONCELOS, J.L.M.; SARTORI, R.; OLIVEIRA, H.N. et al. Reduction in size of the ovulatory follicle reduces subsequent luteal size and pregnancy rate. Theriogenology, v.56, p. $307-314,2001$.

WALKER, W.L.; NEBEL, R.L.; MCGILLIARD, M.L. Time of ovulation relative to mounting activity in dairy cattle. Journal Dairy Science, v.79, p. $1555-1561,1996$. 